\title{
Time-Reversal Division Multiple Access in Multi-path Channels
}

\author{
Feng Han, Yu-Han Yang, Beibei Wang*, Yongle Wu* and K. J. Ray Liu \\ Department of ECE, University of Maryland, College Park, MD, 20742, USA \\ Email: \{hanf, yhyang, kjrliu\}@umd.edu \\ *Qualcomm Inc., San Diego, CA, 92121, USA \\ Email: \{beibeiw, yonglew\}@qualcomm.com
}

\begin{abstract}
Multi-path effect makes high speed broadband communications a very challenging task due to the severe intersymbol interference (ISI). By concentrating energy in both spatial and temporal domains, time-reversal (TR) transmission technique provides a great potential of low-complexity energyefficient communications. In this paper, we develop a new concept of time-reversal division multiple access (TRDMA) as a wireless channel access method based on its high-resolution spatial focusing effect. We propose to use TR structure in a multiuser downlink system over large delay spread channels, where the signals of different users are separated solely by TRDMA. Both the single-transmit-antenna scheme and its enhanced version with multiple-transmit-antenna are developed and evaluated in this paper. We investigate the system performance in terms of the effective SINR and the achievable sum rate of the multi-user system. It is shown in both analytical and simulation results that satisfying performances can be achieved in the proposed TRDMA multi-user downlink system.
\end{abstract}

\section{INTRODUCTION}

The past decade has witnessed an unprecedentedly increasing demand for high speed wireless services, which necessitates the need for future broadband communications. When it comes to broadband, the resolution of perceiving multiple paths increases accordingly. In a rich scattering environment, the adverse multi-path effect makes high speed communications over large delay spread channels a very challenging task, due to the severe ISI . To solve this problem, multi-carrier modulation (e.g. OFDM) and/or complicated equalization are needed [1]-[3] at the receiver to alleviate the ISI. But it results in a prohibitively high complexity for handsets in many wireless applications.

Time-reversal (TR) signal transmission technique can provide a great potential of low-complexity energy-efficient communications, which can make full use of the multi-path environment to focus the signal energy at the intended location. The history of research on time reversal in communications dates back to early 1990's [4]-[8]; however, not much development and interest went beyond the acoustics and ultrasound domains at that time. It was later studied and tested in wireless radio communication systems, especially in Ultra-wideband (UWB) systems [9], [10]. The single-user TR wireless communications consist two phases: the recording phase and the transmission phase. By utilizing channel reciprocity [11], the TR waves can retrace the incoming paths, ending up with a constructive combining in physics and a resulting "spiky" spatial signal-power distribution focused at the intended location [9] [12], as referred to as spatial focusing effect. Also, from a signal processing point of view, in singleuser communications, TR essentially leverages the multi-path channel as a facilitating matched filter computing machine for the intended receiver, and concentrates the signal energy in the time domain as well, as referred to as temporal focusing effect.

In this paper, we consider a multi-user downlink system over multi-path channels, and propose a concept of time-reversal division multiple access (TRDMA) as a wireless channel access method based on the high-resolution spatial focusing effect of time-reversal structure. In principle, the mechanisms of reflection, diffraction and scattering in wireless medium give rise to the uniqueness and independence of the multi-path propagation profile of each communication link [11], which can be exploited to provide spatial selectivity in all existing spatial division multiple access (SDMA) schemes. Compared with conventional antenna-array based beamforming SDMA schemes, time-reversal technique makes full use of a large number of multi-paths and in essence treats each path as a virtual passive antenna that naturally exists and is widely distributed in the environment. Thus, with even just one single transmit antenna, time reversal can potentially achieve very high diversity gain and high-resolution "pin-point" spatial focusing. The high-resolution spatial focusing effect makes the natural multi-path propagation profile into a unique locationspecific signature for each link, as an analogy to the artificial "orthogonal random code" in code division systems.

The proposed TRDMA scheme exploits the uniqueness and independence of location-specific signatures in multipath environment, providing a novel low-cost energy-efficient solution for SDMA. The potential and feasibility of applying time reversal to multi-user UWB communications were validated and supported by the recent antenna-and-propagation experiments in [13], [14], in which the signal transmit power reduction and inter-user interference (IUI) alleviation as a result of spatial focusing effect were tested and justified for one simplified single-bit transmission over a deterministic multi-path ultra-wideband channels. However, there is still a lack of system-level design and performance evaluation of a 
well-functioning TR-based multi-user communication paradig$\mathrm{m}$ in the literature. Motivated by the high-resolution spatial focusing potential of the time-reversal structure and existing experimental measurements, several major generalizations and developments have been made in our proposed TRDMA paradigm to fulfill the challenges of designing a functioning multi-user communication system:

- We propose the concept of TRDMA as an efficient multiuser downlink scheme for wireless multi-path environments, based on the fundamental physics of time reversal.

- We consider a multi-user broadband communication system over multi-path Rayleigh fading channels, and develop a TR-based system where consecutive transmission are supported by taking into account the ISI .

- We define and evaluate a number of system performance metrics, including the focusing gains, the effective SINR at each user, and the achievable sum rate.

- We also extend the SISO (single-input single-output) scheme to a MISO (multiple-input single-output) one, facilitating an enhanced spatial focusing effect and better system performances.

The rest of this paper is organized as follows. In Section II, we introduce the channel model and the proposed TRDMA multi-user downlink system. Then, we analyze the system performance in Section III. An enhanced version with multiple transmit antennas is developed in Section IV. In Section V, simulation results are presented to justify the analytical results and the system performance. Conclusions are drawn in Section VI.

\section{SYSTEM MODEL}

\section{A. Channel Model}

In this paper, we consider a multi-user downlink network over multi-path Rayleigh fading channels. We first look at a SISO case where the base station (BS) and all users are equipped with one single antenna.

The channel impulse response (CIR) of the communication link between the BS and the $i$-th user is modeled as

$$
h_{i}[k]=\sum_{l=0}^{L-1} h_{i, l} \delta[k-l],
$$

where $h_{i}[k]$ is the $k$-th tap of the CIR with length $L$. For each link, we model that $h_{i}[k]$ 's are independent circular symmetric complex Gaussian random variables with zero mean and variance

$$
E\left[\left|h_{i}[k]\right|^{2}\right]=e^{-\frac{k T_{S}}{\sigma_{T}}}, \quad 0 \leqslant k \leqslant L-1
$$

where $T_{S}$ is the sampling period of this system such that $1 / T_{S}$ equals the system bandwidth $B$, and $\sigma_{T}$ is the delay spread [3] of the channel. Due to the two-phase nature of time reversal structure, we assume that the channels are reciprocal, ergodic and blockwise-constant with their tap values remaining fixed during at least one duty cycle, which consists of one pilot transmission in the recording phase and a few data transmissions in the transmission phase, depending how fast the channels vary over time.

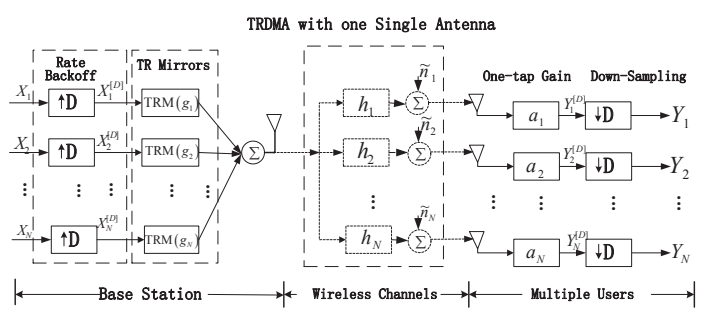

Fig. 1. The diagram of SISO TRDMA multi-user downlink system

We will further assume that the CIRs associated with different users are uncorrelated. While realistic CIRs might not be perfectly uncorrelated, this assumption greatly simplifies the analysis while capturing the essential idea of TRDMA.

\section{B. Phase 1: Recording Phase}

The block diagram of SISO TRDMA downlink system is shown in Fig. 1, in which there are $N$ users receiving statistically independent messages $\left\{X_{1}(k), X_{2}(k), \cdots, X_{N}(k)\right\}$ from the BS, respectively. The time-reversal mirror (TRM) shown in the diagram is a device that can record and time-reverse (and conjugate if complex-valued) the received waveform; and then modulate the time-reversed waveform with input signal by convolving them together. Note that the receivers of interest here are of very low complexity, each with only one tap gain $a_{i}$. The complexity of such a transmitting BS is moderate, but its computational cost is still very low when TR structure becomes the choice.

During the recoding phase, the $N$ intended users first take turns to transmit an impulse signal to the BS (ideally it can be a $\delta$ function, but in practice a modified raise-cosine signal can be a good candidate for limited bandwidth for this purpose [12]). Meanwhile, the TRMs at the BS record the channel response of each communication link and store the timereversed and conjugated version of each channel response for the transmission phase.

For simplicity of analytical derivation, unless otherwise stated, we assume in our theoretical analysis that the waveform recorded by TRM reflects the true values of CIR, ignoring the corruption caused by thermal noise, quantization noise, and nonideal impulse pilot signal.

\section{Phase 2: Transmission Phase}

After the channel recording phase, the system starts its transmission phase. At the BS, each of $\left\{X_{1}, X_{2}, \cdots, X_{N}\right\}$ represents a sequence of information symbols that are complex random variables with zero mean and variance of $P$. We assume that for each $i$ from 1 to $N, X_{i}[k]$ and $X_{i}[l]$ are independent when $k \neq l$. As we mentioned earlier, any two sequences of $\left\{X_{1}, X_{2}, \cdots, X_{N}\right\}$ are also independent in our model. We introduce the rate back-off factor [10] by performing up-sampling and down-sampling with a factor $D$ at the BS and receivers, respectively.

These sequences are first up-sampled by a factor of $D$ at 
the BS, and the $i$-th up-sampled sequence can be expressed as

$$
X_{i}^{[D]}[k]=\left\{\begin{array}{cl}
X_{i}[k / D], & \text { if } k \bmod D=0, \\
0, & \text { if } k \bmod D \neq 0 .
\end{array}\right.
$$

Then the up-sampled sequences are fed into the TRM bank $\left\{g_{1}, g_{2}, \cdots, g_{N}\right\}$, where the output of the $i$-th TRM $g_{i}$ is the convolution of the $i$-th up-sampled sequence $\left\{X_{i}^{[D]}[k]\right\}$ and the TR waveform $\left\{g_{i}[k]\right\}$ as shown in Fig. 1, with

$$
g_{i}[k]=h_{i}^{*}[L-1-k] / \sqrt{E\left[\sum_{l=0}^{L-1}\left|h_{i}[l]\right|^{2}\right]},
$$

the normalized (by the average channel gain) complex conjugate of time-reversed $\left\{h_{i}[k]\right\}$. After that, all the outputs of TRM bank are summed up together, and then the combined signal $\{S[k]\}$ is transmitted into the wireless channels with

$$
S[k]=\sum_{i=1}^{N}\left(X_{i}^{[D]} * g_{i}\right)[k] .
$$

In essence, by convolving the information symbol sequences and the TR waveforms, TRM provides a unique mechanism of embedding the location-specific signature associated with each communication link into the transmitted signal for the intended users.

The signal received at user $i$ is the convolution of $\{S[k]\}$ and $\left\{h_{i}[k]\right\}$, plus a white Gaussian additive noise $\left\{\tilde{n}_{i}[k]\right\}$ with zero mean and variance $\sigma^{2}$. The $i$-th receiver (user $i$ ) simply performs a one-tap gain adjustment to the received signal and then down-samples it with the same factor $D$. The signal before down-sampling can be represented as

$$
Y_{i}^{[D]}[k]=\sum_{j=1}^{N} a_{i}\left(X_{j}^{[D]} * g_{j} * h_{i}\right)[k]+a_{i} \tilde{n}_{i}[k] .
$$

Accordingly, the down-sampled signal $Y_{i}[k]$ is as follows (for simplicity, $L-1$ is assumed to be a multiple of $D$ )

$$
Y_{i}[k]=a_{i} \sum_{j=1}^{N} \sum_{l=0}^{(2 L-2) / D}\left(h_{i} * g_{j}\right)[D l] X_{j}[k-l]+a_{i} n_{i}[k],
$$

where $n_{i}[k]=\tilde{n}_{i}[D k]$, a white Gaussian additive noise with zero mean and variance $\sigma^{2}$.

\section{SyStem PERformance ANALysis}

\section{A. Performance Metric}

In this part, we evaluate the system performances of the proposed TRDMA scheme. Specifically, we investigate the effective signal-to-interference-plus-noise ratio (SINR) at each user in such a downlink scenario.

Note that for $\left\{\left(h_{i} * g_{j}\right)[k]\right\}$, when $k=L-1$ and $i=$ $j$, it corresponds to the maximum-power central peak of the autocorrelation function of the $i$-th CIR, i.e.

$$
\left(h_{i} * g_{i}\right)[L-1]=\sum_{l=0}^{L-1}\left|h_{i}[l]\right|^{2} / \sqrt{E\left[\sum_{l=0}^{L-1}\left|h_{i}[l]\right|^{2}\right]} .
$$

Subject to the constraint of one-tap receivers, the $i$-th receiver is designed to estimate $X_{i}\left[k-\frac{L-1}{D}\right]$ solely based on the observation of $Y_{i}[k]$. Then, the remaining components of $Y_{i}$ can be further categorized into the ISI, the IUI and the noise.
Note that the one-tap gain $a_{i}$ does not affect the effective SINR, we consider it as $a_{i}=1$ without loss of generality, in the subsequent analysis. Given a specific realization of the random CIRs, one can calculate the signal power $P_{S i g}$

$P_{S i g}=E_{X}\left[\left|\left(h_{i} * g_{i}\right)[L-1] X_{i}\left[k-\frac{L-1}{D}\right]\right|^{2}\right]=P\left|\left(h_{i} * g_{i}\right)[L-1]\right|^{2}$,

where $E_{X}[\cdot]$ represents the expectation over $X$. Accordingly, the ISI and IUI can be derived as

$$
\begin{gathered}
P_{I S I}=P \sum_{\substack{l=0 \\
l \neq(L-1) / D}}^{(2 L-2) / D}\left|\left(h_{i} * g_{i}\right)[D l]\right|^{2}, \\
P_{I U I}=P \sum_{\substack{j=1 \\
j \neq i}}^{N} \sum_{l=0}^{(2 L-2) / D}\left|\left(h_{i} * g_{j}\right)[D l]\right|^{2} .
\end{gathered}
$$

The SINR at user $i$ for a given realization of random CIRs can be calculated accordingly with $S I N R=\frac{P_{S i g}}{P_{I S I}+P_{I U I}+\sigma^{2}}$.

\section{B. Average Effective SINR}

We now define the average effective SINR $S I N R_{a v g}$ as

$$
S I N R_{a v g}=\frac{E\left[P_{S i g}\right]}{E\left[P_{I S I}\right]+E\left[P_{I U I}\right]+\sigma^{2}} .
$$

Based on the model assumed in Section II, we average each term in Eqn. (12) over all possible realizations of channels, and obtain the following (after some mathematical work):

$$
\begin{gathered}
E\left[P_{\text {Sig }}\right]=2 P\left(1-e^{-\frac{(L+1) T_{S}}{\sigma_{T}}}\right) /\left(1-e^{-\frac{2 T_{S}}{\sigma_{T}}}\right), \\
E\left[P_{I S I}\right]=\frac{2 P e^{-\frac{T_{S}}{\sigma_{T}}}\left(1-e^{-\frac{(L-2+D) T_{S}}{\sigma_{T}}}\right)}{\left(1-e^{-\frac{D T_{S}}{\sigma_{T}}}\right)\left(1+e^{-\frac{T_{S}}{\sigma_{T}}}\right)}, \\
E\left[P_{I U I}\right]=(N-1) P \\
\times \frac{\left(1+e^{-\frac{D T_{S}}{\sigma_{T}}}\right)\left(1+e^{-\frac{2 L T_{S}}{\sigma_{T}}}\right)-2 e^{-\frac{(L+1) T_{S}}{\sigma_{T}}}\left(1+e^{-\frac{(D-2) T_{S}}{\sigma_{T}}}\right)}{\left(1-e^{-\frac{D T_{S}}{\sigma_{T}}}\right)\left(1+e^{-\frac{T_{S}}{\sigma_{T}}}\right)\left(1-e^{-\frac{L T_{S}}{\sigma_{T}}}\right)} .
\end{gathered}
$$

Then, the average effective SINR $S I N R_{\text {avg }}$ can be calculated accordingly by substituting Eqn. (13), (14) and (15) into Eqn. (12).

\section{TRDMA WITH MULTIPLE TRANSMIT ANTENNAS}

In this part, we introduce a generalized version with multiple transmit antennas at the BS. To maintain low complexity at receivers, we consider a MISO case where the transmitting BS is equipped with $M_{T}$ antennas in comparison to multiple single-antenna users.

In alignment with the basic SISO case, we also assume that paths associated with different antennas are uncorrelated, i.e. $h_{i}^{(m)}[k]$ and $h_{j}^{(w)}[l]$ are uncorrelated for $\forall i, j \in\{1,2, \cdots, N\}$ and $\forall k, l \in\{0,1, \cdots, L-1\}$ when $m \neq w$, where $m, w \in$ $\left\{1,2, \cdots, M_{T}\right\}$ are the indices of the $m$-th and $w$-th antennas at the BS. Let $h_{i}^{(m)}[k]$ denote the $k$-th tap of the CIR for the communication link between user $i$ and the $m$-th antenna of the BS, a circular symmetric complex Gaussian random 


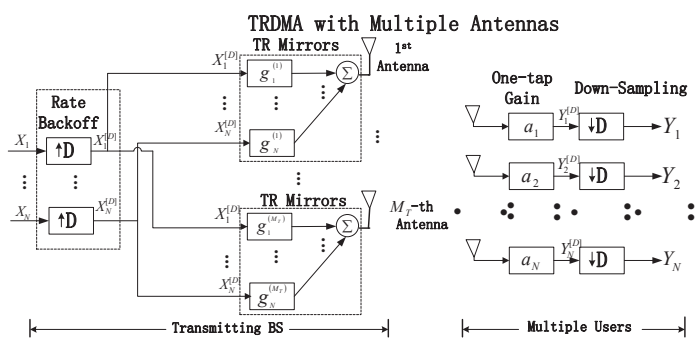

Fig. 2. The diagram of MISO TRDMA multi-user downlink system

variable with zero mean and variance $E\left[\left|h_{i}^{(m)}[k]\right|^{2}\right]=e^{-\frac{k T_{S}}{\sigma_{T}}}$.

For the MISO TRDMA scheme, each antenna at BS plays a role that is similar to the single-antenna BS. The block diagram for the MISO TRDMA is shown in Fig. 2. The TR waveform $\left\{g_{i}^{(m)}[k]\right\}$ is the normalized (by the average gain of MISO channels) complex conjugate of time-reversed $\left\{h_{i}^{(m)}[k]\right\}$, i.e.

$$
g_{i}^{(m)}[k]=h_{i}^{(m) *}[L-1-k] / \sqrt{E\left[M_{T} \sum_{l=0}^{L-1}\left|h_{i}^{(m)}[l]\right|^{2}\right]} .
$$

The resulting received signal at user $i$ can be represented as

$$
Y_{i}[k]=\sum_{j=1}^{N} \sum_{m=1}^{M_{T}} \sum_{l=0}^{\frac{2 L-2}{D}}\left(h_{i}^{(m)} * g_{j}^{(m)}\right)[D l] X_{j}[k-l]+n[k],
$$

based on which the information symbol $X_{i}\left[k-\frac{L-1}{D}\right]$ is estimated at user $i$, and

$$
\begin{gathered}
P_{\text {Sig }}=P\left|\sum_{m=1}^{M_{T}}\left(h_{i}^{(m)} * g_{i}^{(m)}\right)[L-1]\right|^{2}, \\
P_{I S I}=P \sum_{\substack{l=0 \\
l \neq \frac{L}{D}}}^{\frac{2 L-2}{D}}\left|\sum_{m=1}^{M_{T}}\left(h_{i}^{(m)} * g_{i}^{(m)}\right)[D l]\right|^{2}, \\
P_{I U I}=P \sum_{\substack{j=1 \\
j \neq i}}^{\frac{2 L-2}{D}}\left|\sum_{l=0}^{M_{T}}\left(h_{i}^{(m)} * g_{j}^{(m)}\right)[D l]\right|^{2} .
\end{gathered}
$$

With similar mathematical techniques, the average effective SINR for the MISO case at user $i$ is obtained as

$$
S I N R_{a v g}^{(M I S O)}=\frac{E\left[P_{S i g}\right]}{E\left[P_{I S I}\right]+E\left[P_{I U I}\right]+\sigma^{2}},
$$

where

$$
\begin{gathered}
E\left[P_{S i g}\right]=2 P \frac{1-e^{-\frac{(L+1) T_{S}}{\sigma_{T}}}}{1-e^{-\frac{2 T_{S}}{\sigma_{T}}}}+\left(M_{T}-1\right) P \frac{1-e^{-\frac{L T_{S}}{\sigma_{T}}}}{1-e^{-\frac{T_{S}}{\sigma_{T}}}}, \\
E\left[P_{I S I}\right]=2 P \frac{e^{-\frac{T_{S}}{\sigma_{T}}}\left(1-e^{-\frac{(L-2+D) T_{S}}{\sigma_{T}}}\right)}{\left(1-e^{-\frac{D T_{S}}{\sigma_{T}}}\right)\left(1+e^{-\frac{T_{S}}{\sigma_{T}}}\right)}, \\
E\left[\begin{array}{c}
E\left[P_{I U I}\right]=(N-1) P \\
\left(1+e^{-\frac{D T_{S}}{\sigma_{T}}}\right)\left(1+e^{-\frac{2 L T_{S}}{\sigma_{T}}}\right)-2 e^{-\frac{(L+1) T_{S}}{\sigma_{T}}}\left(1+e^{-\frac{(D-2) T_{S}}{\sigma_{T}}}\right)
\end{array}\right.
\end{gathered}
$$

From (23) and (24), one can see that the average interference power maintains as the number of transmit antennas increases, when the normalized TR waveforms are used as shown in
(16). Meanwhile, the second term in (22) demonstrates the enhancement of signal strength and the improved focusing effect as a result of multiple transmit antennas.

\section{NumERiCAL RESUlts}

In this part, we present some numerical results about the performance of the proposed TRDMA multi-user downlink scheme. Unless otherwise stated, simulations shown in this section were performed with $L=257$ and $\sigma_{T}=125 T_{S}$ for the system model. We define a modified SNR per symbol as

$$
\rho=\frac{P}{\sigma^{2}} E\left[\sum_{l=0}^{L-1}\left|h_{i}^{(m)}[l]\right|^{2}\right]=\frac{P}{\sigma^{2}} \frac{1-e^{-\frac{L T_{S}}{\sigma_{T}}}}{1-e^{-\frac{T_{S}}{\sigma_{T}}}},
$$

to rule out the potential multi-path gain in the system model for a fair comparison.

\section{A. Effective SINR}

In this part, we show the average effective SINR as a function of $\rho$ under various system configurations. The SINR curves are obtained according to (12) and (21), and the points marked with "*" are collected from simulation data.

Fig. 3 is plotted with $D=8$ and $N=5$, demonstrating the impact of the number of antennas $M_{T}$ to the effective SINR. From Fig. 3, one can see that approximately $3 \mathrm{~dB}$ gain is attained as $M_{T}$ is doubled within a reasonable range. The impact of the rate back-off to the effective SINR is shown with $N=5, M_{T}=4$ in Fig. 4 . Both analytical formulas and simulation results show that a lager $D$ can effectively reduce ISI and IUI while maintaining the signal power, leading to a higher SINR at each user. In Fig. 5, we investigate the impact of the number of users with $D=8, M_{T}=4$. Due to the existence of IUI, increasing the number of co-existing users will result in higher interference between users. That implies a tradeoff between the network capacity (number of users) and the effective SINR at each user, as indicated in Fig. 5.

\section{B. Achievable Sum Rate}

We evaluate the achievable sum rate of this downlink network using the following equation

$$
R=\frac{1}{B T_{S} \cdot D} \sum_{i=1}^{N} \log _{2}\left(1+S I N R_{i}\right)=\frac{1}{D} \sum_{i=1}^{N} \log _{2}\left(1+S I N R_{i}\right)
$$

where $S I N R_{i}$ denotes the effective SINR at user $i$. In (26), the quantity is divided by $D$, taking into account the rate backoff. We normalize the sum rate with bandwidth $B=1 / T_{S}$, presenting the information rate achieved at unit bandwidth (referred to as spectral efficiency in some cases). Also, note that (26) neglects the overhead caused by the recording phase in each duty cycle, which is valid when the fading channels are not changing very fast; otherwise, when such overhead is not neglectable, one can simply multiply a discount factor $\delta$ to the quantity, where $\delta$ is defined as the ratio of the duration of transmission phase to the entire duty cycle.

We plot this normalized achievable sum rate (omitting the factor $\delta$ ) in Fig. 6 with different system configurations. From both (26) and the numerical results shown in Fig. 6, one can see that the sum rate increases monotonically with the increase 


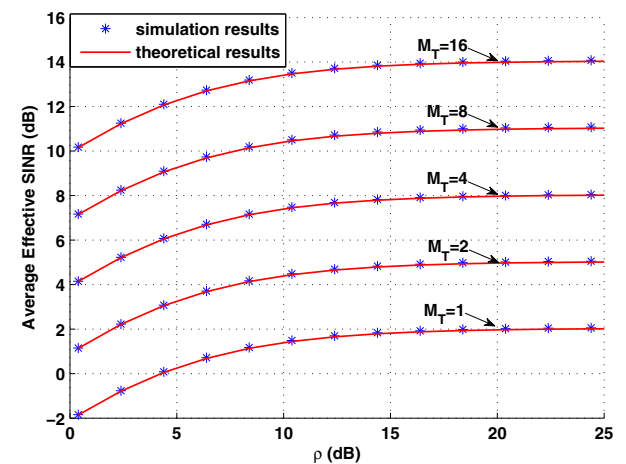

Fig. 3. The impact of the number of antennas when $D=8, N=5$

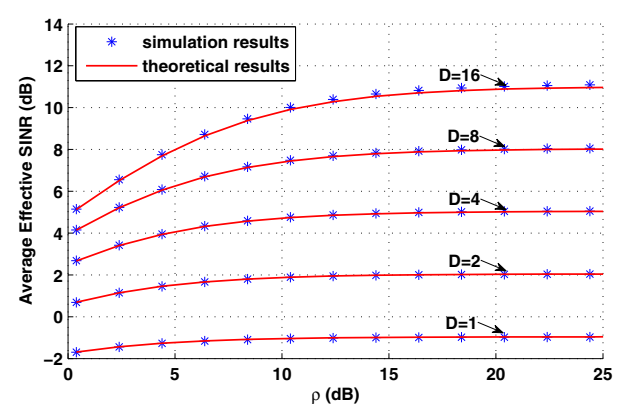

Fig. 4. The impact of the rate back-off factor when $N=5, M_{T}=4$

in $M_{T}$, as a result of improved SINRs achieved by enhanced spatial focusing. The rate back-off also improves SINRs, but incurs penalty of discounting the sum rate by a factor of $D$. Comparatively, the increase in the number of co-existing users $N$ raises the multiplexing order, but results in stronger IUI. Thus, a choice of the pair $(D, N)$ can reveal an engineering tradeoff between the signal quality at each user and the sum rate of this network.

\section{CONCLUSION}

In this paper, we proposed a time-reversal division multiple access scheme for multi-user downlink networks over multipath channels. Such a scheme exploits the nature of multi-path environments, and thus can harvest energy at intended receiv-

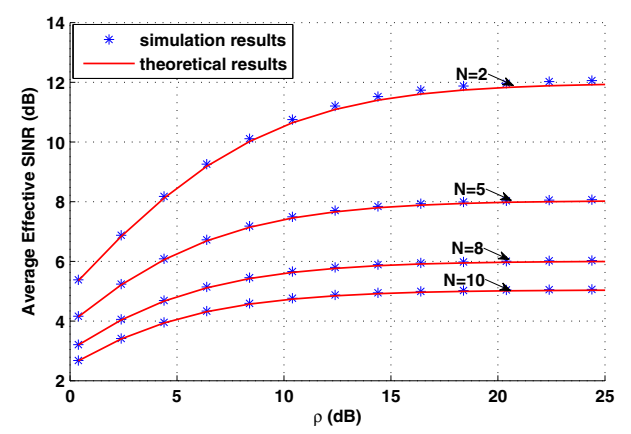

Fig. 5. The impact of the number of users when $D=8, M_{T}=4$

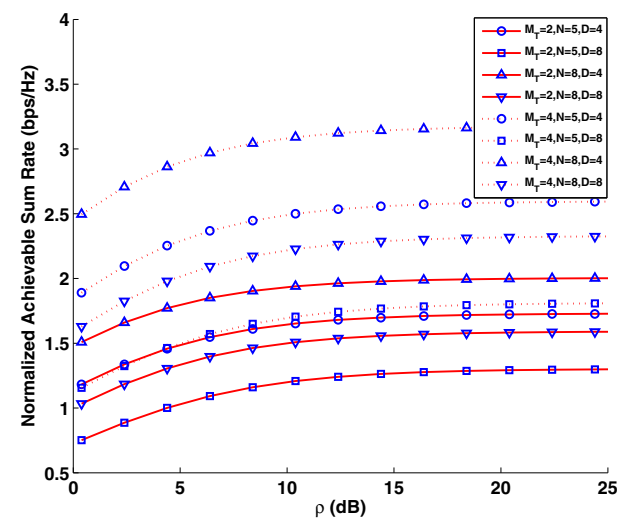

Fig. 6. The normalized achievable sum rate versus $\rho$

ing location and reduce interference among users, thanks to the spatial focusing effect of the TR-structure. We investigated the system performance in terms of the effective SINR and the achievable sum rate, based on the multi-path Rayleigh fading channel model. It was shown in both analysis and simulation that satisfying effective SINR and sum rate can be achieved with reasonable number of transmit antennas and rate back-off in this proposed scheme.

\section{REFERENCES}

[1] J. G. Proakis, Digital Communications, $4^{\text {th }}$ ed., McGraw-Hill, NY, 2001.

[2] G. L. Stuber, Principles of Mobile Communications, $2^{\text {nd }}$ ed., Kluwer, Dordrecht, 2001.

[3] A. J. Goldsmith, Wireless Communication, $1^{\text {st }}$ ed., New York: Cambridge University, 2005.

[4] M. Fink, C. Prada, F. Wu, and D. Cassereau, "Self focusing in inhomogeneous media with time reversal acoustic mirrors," IEEE Ultrasonics Symposium, vol. 1, pp. 681-686, 1989.

[5] C. Prada, F. Wu, and M. Fink, "The iterative time reversal mirror: A solution to self-focusing in the pulse echo mode," J. Acoustic Society of America, vol. 90, pp. 1119-1129, 1991.

[6] M. Fink, "Time reversal of ultrasonic fields. Part I: Basic principles," IEEE Transactions on Ultrasonic, Ferroelectronic, and Frequency Control, vol. 39, pp. 555-566, September 1992.

[7] C. Dorme and M. Fink, "Focusing in Transmit-receive mode through inhomogeneous media: The time reversal matched filter approach," $J$. Acoustic Society of America, vol. 98, pp. 1155-1162, Aug. 1995.

[8] A. Derode, P. Roux, and M. Fink, "Robust acoustic time reversal with high-order multiple scattering," Phys. Rev. Letters, vol. 75, pp. 42064209, 1995.

[9] S. M. Emami, J. Hansen, A.D. Kim,G. Papanicolaou and A.J. Paulraj, "Predicted time reversal performance in wireless communications," IEEE Communications Letters, 2004.

[10] M. Emami, M. Vu, J. Hansen, A.J. Paulraj, and G. Papanicolaou "Matched filtering with rate back-off for low complexity communications in very large delay spread channels," Proc. 38th Asilomar Conf. Signals, Syst. Comput., vol. 1, pp. 218-222, Nov. 2004.

[11] K. F. Sander, G. A. L. Reed, Transmission and propagation of electromagnetic waves, $2^{\text {nd }}$ ed., Cambridge University Press, 1986.

[12] B. Wang, Y. Wu, F. Han, Y. H. Yang, and K. J. R. Liu, "Green wireless communications: a time-reversal paradigm," submitted to IEEE JSAC, Special Issue on Energy-Efficient Wireless Communications.

[13] H. T. Nguyen, I. Z. Kovacs, P. C. F. Eggers "A time reversal transmission approach for multiuser UWB communications," IEEE Transactions on Antennas and Propagation, vol. 54, No. 11,pp. 3216-3224, Nov. 2006

[14] H. T. Nguyen, "Partial one bit time reversal for UWB impulse radio multi-user communications," International Conference on Communications and Electronics, pp. 246-251, June, 2008 